\title{
Proviral DNA as a Target for HIV-1 Resistance Analysis
}

\author{
Nadine Lübke ${ }^{a}$ Veronica Di Cristanziano ${ }^{a}$ Saleta Sierra ${ }^{a}$ Elena Knops ${ }^{\text {a }}$ \\ Eugen Schülter $^{a}$ Björn Jensen ${ }^{c}$ Mark Oette ${ }^{b}$ Thomas Lengauer $^{d}$ \\ Rolf Kaiser $^{\mathrm{a}}$ on behalf of the Resina Study Group \\ ${ }^{a}$ Institute of Virology, University of Cologne, and b Department of Gastroenterology, KH. d. Augustinerinnen, \\ Cologne, ' Department of Gastroenterology, Hepatology and Infectiology, University of Düsseldorf, Düsseldorf, and \\ dThe Computational Biology and Applied Algorithmics Department, Max Planck Institute for Informatics, \\ Saarbrücken, Germany
}

\section{Key Words}

HIV-1 resistance testing - Proviral DNA - Cellular reservoirs .

Low-level viremia $\cdot$ Drug resistance

\begin{abstract}
Background: Resistance analysis from viral RNA is restricted to detectable viral load. Therefore, analysis from proviral DNA could help in cases with low-level or suppressed viremia. Methods: Viral plasma RNA and the corresponding cellular proviral DNA of 78 EDTA samples from 48 therapy-naïve (TN) and 30 therapy-experienced (TE) HIV-1-infected patients were isolated and analyzed for their resistance profiles in the protease and reverse transcriptase genes. Results: Overall, 175 drug-resistance mutations (DRMs) were detected in 25/30 TE (83.3\%) and 5/48 TN (10.4\%) samples. The TE patients displayed a mean number of 6.68 DRMs in RNA and 5.20 in DNA. In the TN patients, a mean of 0.8 DRMs was found in RNA and 1.0 in DNA; $75 \%$ of the DRMs were detected in RNA and DNA simultaneously. In the TE samples, 76\% of the DRMs were detected simultaneously in RNA and DNA, $23 \%$ exclusively in RNA and $1 \%$ in DNA only. The TN samples revealed a significantly higher frequency of DRMs in DNA than in RNA. Conclusions: Proviral DNA resistance testing provides additional resistance information for TN patients. It
\end{abstract}

is also a reliable alternative for TE patients with unsuccessful RNA testing and can provide valuable information when no records are available.

\section{Introduction}

The European guidelines for the clinical use of HIV drug resistance testing recommend the genotypic analysis of protease (PR) and reverse transcriptase (RT) for drugnaïve patients with acute and chronic infection as well as monitoring the efficacy of antiretroviral therapy (ART) of $\mathrm{HIV}$-infected patients in the event of virologic failure [1].

According to the German-Austrian guidelines for the management of HIV infection (http://www.daignet.de/ site-content/hiv-therapie/leitlinien-1), therapy failure is defined as having a viral load of $>50$ copies $/ \mathrm{ml}$, so genotypic drug resistance analysis should be performed at the time of low-level viremia (LLV). These recommendations are also strengthened by the fact that persistent LLV is associated with an increased risk of virologic failure [2]. HIV drug resistance mutations (DRMs) detected during LLV are strongly associated with subsequent virologic failure, thus patients with resistance during LLV have a

\section{KARGER 125}

(c) 2015 S. Karger AG, Basel

0300-5526/15/0583-0184\$39.50/0

E-Mail karger@karger.com

www.karger.com/int
Dr. Nadine Lübke

Institute of Virology, University of Cologne

Fürst-Pückler-Strasse 56

DE-50935 Köln (Germany)

E-Mail nadine.luebke@uk-koeln.de 
higher risk of therapy failure $[3,4]$. Furthermore, almost $50 \%$ of ART-treated patients with LLV have an increased probability of accumulation of additional DRMs, which is linked to the number of active drugs. The amount of fully active drugs and the duration of LLV are both predictive of emerging resistance [5].

HIV infects long-lived cells, so the history of genotypes remains archived $[6,7]$. Therefore, wild-type or resistant variants acquired due to transmitted resistance or during the treatment history of the patient are unlikely to completely disappear from the body with or without currently available drug treatment. Thus, viral variants, including circulating drug-resistant strains selected during ART, appear in the latent reservoir as minor variants [810]. For the detection of such minor variants, the analysis of proviral DNA can be a useful technique [11]. This analysis can also be helpful in clinical practice in cases of planned therapy switch in suppressed HIV-1-infected patients with good virologic control [12-14].

To study the degree of information in proviral HIV DNA with respect to resistance, we compared the resistance information of HIV PR and RT in viral RNA and proviral DNA detected by standard genotypic resistance analysis in samples of therapy-naïve (TN) and therapyexperienced (TE) HIV-1-infected patients.

\section{Materials and Methods}

Viral Samples

Eighty HIV-1 samples were randomly selected for this study. They were obtained from 80 RESINA cohort patients treated in cooperating HIV centers in North Rhine-Westphalia, Germany. There were $50 \mathrm{TN}$ patients and $30 \mathrm{TE}$ patients at the time of sample collection.

\section{Genotypic Resistance Analysis}

The viral RNA and the corresponding proviral DNA of the 80 EDTA samples were isolated, amplified, sequenced and analyzed for resistance in the PR and RT genes.

Viral RNA from $500 \mu \mathrm{l}$ plasma and proviral DNA from $100 \mu \mathrm{l}$ buffy coat were isolated automatically using the MagNA Pure ${ }^{\mathrm{TM}} \mathrm{LC}$ total nucleic acid isolation kit (large volume) with the MagNA Pure LC System (Roche Diagnostics).

RT-PCR and nested PCR were performed as previously described $[15,16]$. For RT-PCR, we used the primers PRRT-nonB-F: 5'-GCTACACTAGAAGAAATGATGACAGCATG-3' (nt 1,3561,384 in HXB2) and 3532a: 5'-TTCTGCTATTAAGTCTTTTGATGGGTCA-3. For nested PCR, we used the primers 2001s: 5'-TGCAGGGCCCCTAGGAAAAAGGGCTGTT-3' and 3454as: 5'-AGTGCTAGCTCTGCTTCTTCTGTTAGTGGTA-3'. PCR purification was performed via ExoSap described by Sierra 2011 and sequencing with the ViroSeq HIV genotyping kit v2.0 (Applied Biosystems).

Proviral Resistance Analysis
Table 1. Detection rate of DRMs in viral RNA and proviral DNA

\begin{tabular}{llll}
\hline & Viral RNA & Proviral DNA & p value \\
\hline Total $(\mathrm{n}=30)$ & 171 & 135 & \\
& $5.70 \pm 4.79$ & $4.50 \pm 4.58$ & 0.3311 \\
$\operatorname{PR}(\mathrm{n}=9)$ & 28 & 22 & \\
$\mathrm{RT}(\mathrm{n}=30)$ & $3.11 \pm 2.26$ & $2.44 \pm 1.59$ & 0.5452 \\
& 143 & 113 & \\
$\mathrm{TN}(\mathrm{n}=5)$ & $4.77 \pm 3.39$ & $3.77 \pm 3.34$ & 0.2564 \\
$\mathrm{TE}(\mathrm{n}=25)$ & 4 & 5 & \\
& $0.80 \pm 0.84$ & $1.00 \pm 0.00$ & 0.6089 \\
& 167 & 130 & \\
& $6.68 \pm 4.65$ & $5.20 \pm 4.72$ & 0.2756 \\
\hline
\end{tabular}

Comparison of DRMs present in viral plasma RNA and proviral cellular DNA in the PR and RT genes in TN and TE patients. Values show the number of DRMs and the mean \pm SD for each category. $\mathrm{p}<0.05$ was considered significant.

The sequences generated on an ABI $3130 \mathrm{XL}$ sequencer were aligned with the HIV-1 subtype B reference strain HXB2. DRMs in the PR and RT genes were identified according to the 2014 International Aids Society USA resistance list (www.iasusa.org). Minor PR inhibitor mutations with polymorphic characteristics were not considered in this analysis.

\section{Statistical Analysis}

The comparison of quantitative variables was calculated by means of the Student t test (http://www.graphpad.com/quickcalcs/ ttest $1 /$ ?Format=SD) and categorical variables with the Fisher exact test (http://vassarstats.net/tab $2 \times 2 . h t m l$ ). The significance level was defined as $\mathrm{p}<0.05$.

\section{Results}

The 80 randomly collected samples of the RESINA cohort consisted of 50 from TN patients and 30 from TE patients. The median viral load of the TN samples was 102,982 copies/ml (range 50-1,929,370) and that of the TE samples was 10,208 copies/ml (range 425-311,700). The mean minimal duration of infection in the cohort was 2 years (range $0-8$ ) for TN patients and 12 years (range 1-23) for TE patients.

Seventy-eight of the 80 tested samples (48 from TN and 30 from TE patients) provided amplicons of the PR and RT genes of both viral RNA and proviral DNA, which could then be analyzed. In total, we detected 175 different DRMs in 30/78 samples in the PR or RT genes or in both. One hundred and seventy-one DRMs were detected in the viral RNA and 135 in the proviral DNA (table 1). Thus, the mean number of total pol gene mutations was 


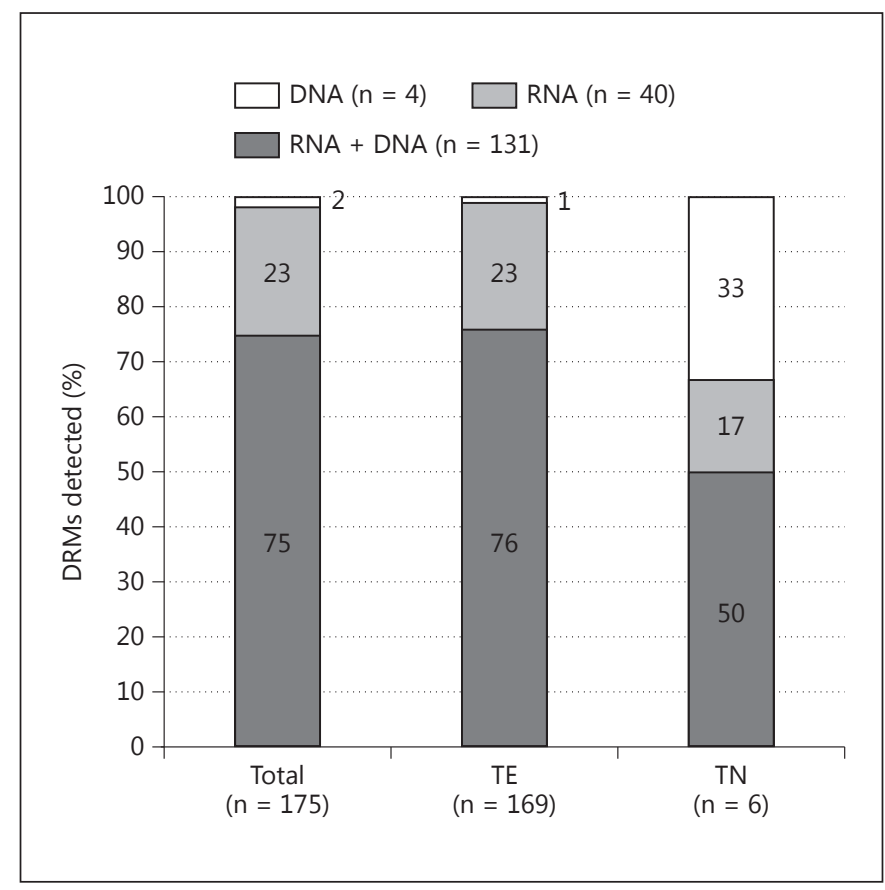

Fig. 1. DRMs detected in viral RNA and proviral DNA in all analyzed samples (Total) and in samples from TE and TN patients.

higher in viral RNA $(5.70 \pm 4.79)$ than in proviral DNA $(4.50 \pm 4.58$; table 1$)$, but did not reach statistical significance $(\mathrm{p}=0.3311)$. Regarding the particular gene regions, a mean \pm SD of $4.77 \pm 3.39 \mathrm{RT}$ and $3.11 \pm 2.26 \mathrm{PR}$ mutations were detected in viral RNA, and $3.77 \pm 3.34 \mathrm{RT}$ and $2.44 \pm 1.59$ PR mutations in proviral DNA (table 1).

When considering the therapy background, the samples from the TE patients, as expected, presented a higher rate of mutations (169 vs. 6; fig. 1). Overall, DRMs were detected in $83.3 \%(25 / 30)$ of the positive samples as opposed to in $10.4 \%(5 / 48)$ of the samples from the TN patients (table 1). The greater mean number of DRMs in viral RNA $(6.68 \pm 4.65)$ compared to in proviral DNA $(5.20 \pm 4.72)$ in the TE patients was not observed in the TN patients, who displayed $0.8 \pm 0.84$ mutations in viral RNA and $1.0 \pm 0.0$ mutations in proviral DNA.

Overall, most DRMs (75\%) were detected in the RNA and DNA simultaneously, while $23 \%$ were found exclusively in viral RNA and $2 \%$ in proviral DNA only (fig. 1). The samples from the TE patients presented $76 \%$ of the DRMs in both RNA and DNA, 23\% exclusively in RNA and $1 \%$ in DNA only. In contrast, the distribution of the DRMs in RNA and DNA found in the samples of TN patients revealed a significantly higher frequency of DRMs in proviral DNA $(33 \% ; \mathrm{p}=0.006)$.
Considering the drug classes, the majority of detected DRMs were nucleoside reverse transcriptase inhibitor (NRTI) mutations $(46 \%, \mathrm{n}=141)$, followed by non-NRTI (NNRTI) mutations $(38 \%, \mathrm{n}=115)$ and PR inhibitor mutations $(16 \%, \mathrm{n}=50)$. In total, 34 different resistance-associated positions were affected, 22 in the RT and 12 in the PR gene (fig. 2). Only 5 DRMs were more frequent in proviral DNA, with the substitutions at positions 74,100 and 230 in the RT and at positions 32 and 47 in the PR gene. In summary, the frequency of DRMs was predominantly higher in the viral RNA genotypes.

\section{Discussion}

At present, the European guidelines for the clinical use of HIV drug resistance testing recommend resistance determination in specific situations, e.g. at the start of ART, at treatment switch or when considering a treatment simplification. HIV-1 resistance testing has been routinely performed from plasma samples, and the European guidelines do not comment on resistance analysis of peripheral blood mononuclear cell-derived proviral DNA. This constitutes a problem for the analysis of samples from patients with LLV or undetectable viremia. Resistant HIV strains acquired at the time of primary infection or selected when ART has failed should be archived intracellularly as proviral DNA. Therefore, resistance analysis from this source may be useful for patients with low viral loads, but also for TE patients without records of previous therapies and resistance tests [11].

We compared the resistance profile from viral RNA and proviral DNA extracted from the very same blood samples in order to determine the informative value of proviral DNA as a basis for HIV resistance testing. PR and RT regions from TN and TE patients were analyzed; 30 samples displayed DRMs either in RNA, DNA or both. Although this analysis revealed an overall higher frequency of DRMs in viral RNA than in proviral DNA, 75\% of the DRMs were detected in both materials. Similar results were published in a study by Banks et al. [17], comparing the pol genotypes of the circulating viral RNA and proviral DNA in the peripheral blood mononuclear cells in 32 blood samples of 25 subtype $\mathrm{C}$-infected patients receiving ART. They also reported similar mutation patterns in viral RNA and proviral DNA, with unique mutations in $>50 \%$ of cases.

However, a subanalysis of our studied samples presented different detection rates of DRMs in viral RNA and proviral DNA in relation to therapy experience. In 
Fig. 2. Detected DRMs in viral RNA and proviral DNA with regard to drug classes and amino acid positions. Detected NRTI (a), NNRTI (b) and PR inhibitor (c) resistance-associated mutations in viral RNA (dark grey) and proviral DNA (light grey). Codons with detected amino acid substitutions are indicated on the $\mathrm{x}$-axis and the percentage of samples presenting mutations are indicated on the $y$-axis.
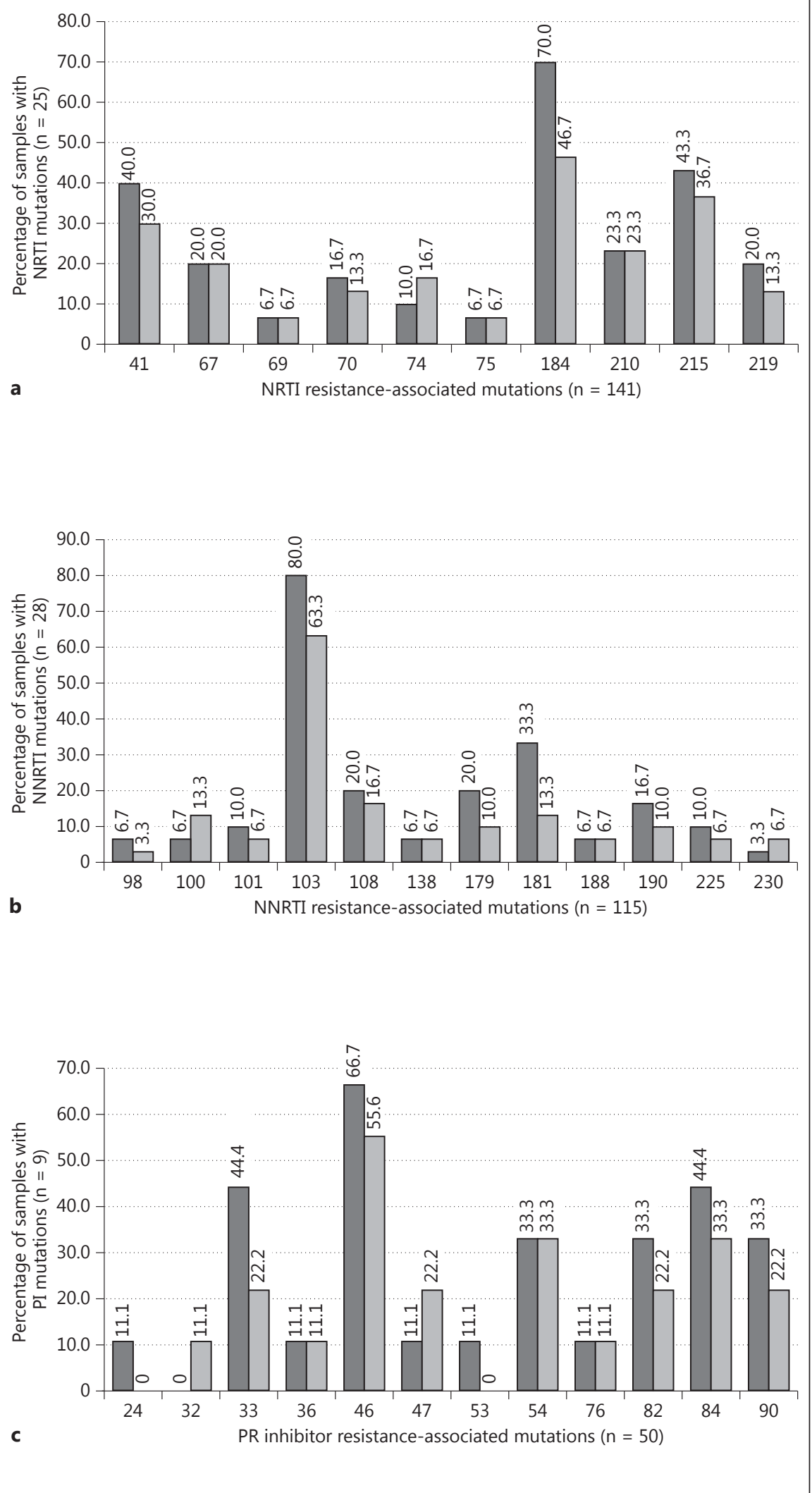
the samples from the TE patients, the majority of DRMs appeared in viral RNA (99\%) whereas those from the TN patients displayed a significantly higher proportion of DRMs in proviral DNA (33\%). These data are in line with other published studies investigating information on resistance in viral RNA and proviral DNA of identical blood samples [17-22].

The differences between TN and TE patients with regard to the DRMs detected in proviral DNA and viral RNA can be explained by the kinetics of transmitted resistance, as it has been found that the amount of resistant variants correlates with the time they take to replicate [10]. While viruses in TN patients have not been exposed to any drug pressure, the transmitted viruses can replicate and uninhibitedly fill the cellular reservoirs, leading to a higher frequency of archived drug-resistant strains. If patients are infected with drug-resistant strains, the replication of the resistant viruses is not in competition with wild-type strains characterized by a higher replication capacity, leading to a higher frequency of archived drugresistant strains. In contrast, in TE patients, viruses with on-treatment-selected DRMs are in competition to wildtype strains, resulting in a comparatively shorter period of replication and a smaller quantity of resistant viruses entering the latent reservoir.

Although our data are limited due to the sample size, the genotyping of HIV proviral DNA proved to be pos- sible and provided useful information. PR and RT DRMs were detected in viral RNA and proviral DNA simultaneously. In addition, the resistance analysis of proviral DNA provided information about transmitted drug resistance in TN patients, and this could lead to improvements in the surveillance of drug resistance for these individuals. In TE patients, more information on resistance was obtained from the viral RNA. However, resistance testing of proviral DNA could provide valuable additional information in cases of an unsuccessful RNA resistance analysis due to LLV or when no historic resistance data are available.

In summary, the combined analysis of viral plasma RNA and peripheral blood mononuclear cell-derived DNA provided complementary information on resistance, representing a meaningful approach for HIV resistance analysis. In addition, proviral DNA testing offers an alternative opportunity when RNA testing is unsuccessful.

\section{Acknowledgements}

The authors thank Dörte Hammerschmidt for invaluable help in sample processing, Claudia Müller for collecting patients' therapy history and Eugen Schülter for database management. We also thank all patients and the treating physicians who contributed to the RESINA Study.

\section{References}

1 Vandamme AM, Camacho RJ, CeccheriniSilberstein F, de Luca A, Palmisano L, Paraskevis D, Paredes R, Poljak M, Schmit JC, Soriano $\mathrm{V}$, Walter $\mathrm{H}$, Sonnerborg A; European HIV Drug Resistance Guidelines Panel: European recommendations for the clinical use of HIV drug resistance testing: 2011 update. AIDS Rev 2011;13:77-108.

-2 Laprise C, de Pokomandy A, Baril JG, Dufresne $\mathrm{S}$, Trottier $\mathrm{H}$ : Virologic failure following persistent low-level viremia in a cohort of HIV-positive patients: results from 12 years of observation. Clin Infect Dis 2013;57:1489-1496.

3 Gonzalez-Serna A, Min JE, Woods C, Chan D, Lima VD, Montaner JS, Harrigan PR, Swenson LC: Performance of HIV-1 drug resistance testing at low-level viremia and its ability to predict future virologic outcomes and viral evolution in treatment-naive individuals. Clin Infect Dis 2014;58:1165-1173.

-4 Swenson LC, Min JE, Woods CK, Cai E, Li JZ, Montaner JS, Harrigan PR, Gonzalez-Serna A: HIV drug resistance detected during low- level viraemia is associated with subsequent virologic failure. AIDS 2014;28:1125-1134.

5 Li JZ, Gallien S, Do TD, Martin JN, Deeks S, Kuritzkes DR, Hatano H: Prevalence and significance of HIV-1 drug resistance mutations among patients on antiretroviral therapy with detectable low-level viremia. Antimicrob Agents Chemother 2012;56:5998-6000.

6 Siliciano JD, Kajdas J, Finzi D, Quinn TC, Chadwick K, Margolick JB, Kovacs C, Gange SJ, Siliciano RF: Long-term follow-up studies confirm the stability of the latent reservoir for HIV-1 in resting CD4+ T cells. Nat Med 2003; 9:727-728.

7 Strain MC, Gunthard HF, Havlir DV, Ignacio CC, Smith DM, Leigh-Brown AJ, Macaranas TR, Lam RY, Daly OA, Fischer M, Opravil M, Levine $\mathrm{H}$, Bacheler L, Spina CA, Richman DD, Wong JK: Heterogeneous clearance rates of long-lived lymphocytes infected with HIV: intrinsic stability predicts lifelong persistence. Proc Natl Acad Sci USA 2003;100: 4819-4824.
8 Lambotte O, Chaix ML, Gubler B, Nasreddine N, Wallon C, Goujard C, Rouzioux C, Taoufik Y, Delfraissy JF: The lymphocyte HIV reservoir in patients on long-term HAART is a memory of virus evolution. AIDS 2004;18: 1147-1158.

-9 Turriziani O, Andreoni M, Antonelli G: Resistant viral variants in cellular reservoirs of human immunodeficiency virus infection. Clin Microbiol Infect 2010;16:15181524.

10 Verhofstede C, Noe A, Demecheleer E, De Cabooter N, Van Wanzeele F, Van Der Gucht B, Vogelaers D, Plum J: Drug-resistant variants that evolve during nonsuppressive therapy persist in HIV-1-infected peripheral blood mononuclear cells after long-term highly active antiretroviral therapy. J Acquir Immune Defic Syndr 2004;35:473-483.

11 Delgado R: Detection of resistance mutations in proviral DNA in HIV-1 infection. Enferm Infecc Microbiol Clin 2013;31(suppl 1):3539. 
12 De Castro N, Braun J, Charreau I, Pialoux G, Cotte L, Katlama C, Raffi F, Weiss L, Meynard JL, Yazdanpanah Y, Delaugerre C, MadelaineChambrin I, Aboulker JP, Molina JM, E.A.s. group: Switch from enfuvirtide to raltegravir in virologically suppressed multidrug-resistant HIV-1-infected patients: a randomized openlabel trial. Clin Infect Dis 2009;49:1259-1267.

13 Delaugerre C, Braun J, Charreau I, Delarue S, Nere ML, de Castro N, May T, Marchou B, Simon F, Molina JM, Aboulker JP, A.E.s. group: Comparison of resistance mutation patterns in historical plasma HIV RNA genotypes with those in current proviral HIV DNA genotypes among extensively treated patients with suppressed replication. HIV Med 2012; 13:517-525.

14 Palmisano L, Galluzzo CM, Giuliano M: The importance of testing genotypic resistance in proviral DNA of patients fully responding to highly active antiretroviral therapy. J Acquir Immune Defic Syndr 2009;51:233-234.

15 Balduin M, Sierra S, Daumer MP, Rockstroh JK, Oette M, Fatkenheuer G, Kupfer B, Beerenwinkel N, Hoffmann D, Selbig J, Pfister
HJ, Kaiser R: Evolution of HIV resistance during treatment interruption in experienced patients and after restarting a new therapy. J Clin Virol 2005;34:277-287.

16 Verheyen J, Litau E, Sing T, Däumer M, Balduin M, Oette M, Fätkenheuer G, Rockstroh JK, Schuldenzucker U, Hoffmann D, Pfister H, Kaiser R: Compensatory mutations at the HIV cleavage sites p7/p1 and p1/p6-gag in therapy-naive and therapy-experienced patients. Antiviral Ther 2006;11:879-887.

17 Banks L, Gholamin S, White L, Zijenah E, Katzenstein DA: Comparing Peripheral blood mononuclear cell DNA and circulating plasma viral RNA pol genotypes of subtype C HIV-1. J AIDS Clin Res 2012;3:141-147.

18 Bon I, Alessandrini F, Borderi M, Gorini R, Re MC: Analysis of HIV-1 drug-resistant variants in plasma and peripheral blood mononuclear cells from untreated individuals: implications for clinical management. New Microbiol 2007;30:313-317.

19 Bon I, Gibellini D, Borderi M, Alessandrini F, Vitone F, Schiavone P, Re MC: Genotypic resistance in plasma and peripheral blood lym- phocytes in a group of naive HIV-1 patients. J Clin Virol 2007;38:313-320.

20 Ghosn J, Pellegrin I, Goujard C, Deveau C, Viard JP, Galimand J, Harzic M, Tamalet C, Meyer L, Rouzioux C, Chaix ML; French PRIMO Cohort Study Group (ANRS CO 06): HIV-1 resistant strains acquired at the time of primary infection massively fuel the cellular reservoir and persist for lengthy periods of time. AIDS 2006;20:159-170.

-21 Kabamba-Mukadi B, Duquenne A, Henrivaux $\mathrm{P}$, Musuamba F, Ruelle J, Yombi JC, Bodeus M, Vandercam B, Goubau P: HIV-1 proviral resistance mutations: usefulness in clinical practice. HIV Med 2010;11:483-492.

-22 Parisi SG, Boldrin C, Cruciani M, Nicolini G, Cerbaro I, Manfrin V, Dal Bello F, Franchin E, Franzetti M, Rossi MC, Cattelan AM, Romano L, Zazzi M, Andreoni M, Palu G: Both human immunodeficiency virus cellular DNA sequencing and plasma RNA sequencing are useful for detection of drug resistance mutations in blood samples from antiretroviral-drug-naive patients. J Clin Microbiol 2007;45:1783-1788. 